\title{
Conformal symmetry of gravity and the cosmological constant problem
}

\author{
Mariano Cadon* \\ Dipartimento di Fisica, Università di Cagliari, and INFN sezione di Cagliari, \\ Cittadella Universitaria 09042 Monserrato, ITALY
}

\begin{abstract}
In absence of matter Einstein gravity with a cosmological constant $\Lambda$ can be formulated as a scale-free theory depending only on the dimensionless coupling constant $G \Lambda$, where $G$ is Newton constant. We derive the conformal field theory (CFT) and its improved stress-energy tensor that describe the dynamics of conformally flat perturbations of the metric. The CFT has the form of a constrained $\lambda \phi^{4}$ field theory. In the cosmological framework the model describes the usual Friedmann-Robertson-Walker flat universe. The conformal symmetry of the gravity sector is broken by coupling with matter. The dimensional coupling constants $G$ and $\Lambda$ are introduced by different terms in this coupling. If the vacuum of quantum matter fields respects the symmetry of the gravity sector, the vacuum energy has to be zero and the "physical" cosmological constant is generated by the coupling of gravity with matter. This could explain the tiny value of the observed energy density driving the accelerating expansion of the universe.
\end{abstract}

The cosmological constant problem (see for reviews 1, 2, 3, 4, 5, 6]) arises in the interplay between particle physics and cosmology. The problem is originated by the attempt to explain the accelerating expansion of the universe, which emerges from the recent remarkable observational discoveries [7, 8, 9, 10, 11, 12, 13, 14], in terms of fundamental laws of microphysics. The observational data can be explained introducing a cosmological constant $\Lambda$ ( a constant curvature term) in the Einstein equations corresponding to a energy density $\rho^{(o b s)} \sim\left(10^{-3} \mathrm{eV}\right)^{4}$ (Unless explicitly stated, throughout this paper we will use natural units). On the other hand, if we identify this energy density with the vacuum energy of ordinary particle physics and believe general relativity (GR) to hold up to Planck scale, we expect $\rho^{(\text {guess })} \sim 10^{120} \rho^{(o b s)}$. We are therefore faced with the following problems: $a$ ) Why is the observed energy density so tiny with respect to that expected from microphysics? b) Why is $\rho$ not exactly equal to zero? 26]

During the last two decades many proposal have been put forward to solve the cosmological constant problem (see e.g. Ref. [1, 2, 3, 4, 5, [6]): symmetry principles, environmental selection, back-reaction, breaking down of general relativity, large extra dimensions, dynamical dark energy. Despite these efforts, none of the proposal can be considered satisfactory. It seems by now evident that the origin of the cosmological constant problem is a very subtle conspiracy between physics at short and large scales. If the energy density $\rho^{(o b s)}$ is originated by a local quantum field theory, it has to be associated to a length scale $L_{s} \sim\left(\hbar / \rho^{(o b s)} c\right)^{1 / 4} \sim 10^{-2} \mathrm{~cm}$ (note that we have reinstated units). This in turn implies the emergence of new physics already at length scales $L_{s}$, which is very unlikely, although not completely ruled out by the experiments. Any modification of the Einstein action - either by short-distance terms (e.g coming from string theory) or by large extra dimensions - are strongly constrained because they are not allowed to modify the gravitational interaction in the range millimeters-astronomical distances. The tiny value of $\rho^{(o b s)}$ could be also traced back to an infrared modification of general relativity at length scales $L_{S} \sim\left(c^{2} / G \rho^{(o b s)}\right)^{1 / 2} \sim 10^{29} \mathrm{~cm}$. But in this case, it is very difficult to understand how the physics at those scales could prevent the vacuum energy density of quantum fields to become comparable with $\rho^{\text {(guess) }}$.

In view of this very intricate situation a proper understanding of the role played by the cosmological constant in the Einstein theory of gravity may be very useful and indicate promising directions for solving the problem. The key idea, which will be the starting point of this paper, is to question the standard geometrical interpretation of $\Lambda$ and to interpret it from a "field theoretical" point of view as a coupling constant. This approach has been first proposed by Polyakov [15] and more recently in Ref. [16]. It has been noted that the conformal mode of Einstein gravity can be described by a $\lambda \phi^{4}$ field theory [15, 16]. It has been also proposed that the quantum triviality of the $\lambda \phi^{4}$ theory could be responsible for infrared screening, driving to zero the value of the cosmological constant [15, 16].

In this paper we will elaborate further on this idea. We will show that in the absence of matter Einstein gravity with a cosmological constant is a scale-free theory. We derive the conformal field theory (CFT) in four dimensions (4D) and its improved stress-energy tensor that describe the dynamics of conformally flat perturbations of the metric. The CFT has the form of a constrained $\lambda \phi^{4}$ field theory. In the cosmological framework the theory describes the usual Friedmann-Robertson-Walker flat universe. The conformal symmetry of the gravity sector is broken by coupling with matter. The coupling constants $G$ and $\Lambda$ are introduced by different terms in this coupling. If the vacuum of matter fields respects the symmetry of the gravity sector, the vacuum energy has to be zero and the "physical" cosmological constant is generated by the coupling of gravity with matter. 
In the Einstein theory of gravity, described by the action

$$
S=\frac{1}{16 \pi G} \int d^{4} x \sqrt{-g}(R-2 \Lambda),
$$

$\Lambda$ appears as a free parameter, which is usually interpreted, geometrically, as a constant curvature term of the spacetime independent from the presence of matter sources. This geometrical interpretation is, from the physical point of view, rather unsatisfactory. First, if the spacetime has a curvature in absence of matter-energy sources, what is the physical origin of the curvature? Second, in the cosmological framework the term looses its geometrical interpretation and becomes a (rather exotic) form of matter, with constant density and equation of state $p=-\rho$. Third, the cosmological constant problem amounts to explain why the effective cosmological constant $\Lambda_{\text {eff }}=\Lambda-8 \pi G\left\langle\rho_{M}\right\rangle$ is so close to zero. But the cancellation required here is rather unnatural. It requires to sum a free parameter with something determined by the matter sources to produce a small number.

The above difficulties do not arise if we forget the geometrical meaning of $\Lambda$ and interpret $\Lambda$ and $G$ on equal footing, as coupling constants. The second step is to use standard dimensional analysis (see for instance Ref. [17] for the case of gravity with zero cosmological constant) to realize that the gravitational field $g_{\mu \nu}$ is not canonically defined. In fact its group dimensionality, which characterizes the behavior under dilatations, is -2 , whereas its engineering dimensionality is 0 . A canonical defined metric tensor is obtained by the redefinition

$$
g_{\mu \nu} \rightarrow \frac{g_{\mu \nu}}{\lambda^{2}}
$$

where $\Lambda=3 \lambda^{2}$. With the field redefinition (2) the Einstein action (1) becomes scale-free and depends only on the dimensionless coupling $\alpha_{0}=G \lambda^{2}$

$$
S=\frac{1}{16 \pi \alpha_{0}} \int d^{4} x \sqrt{-g}(R-6) .
$$

For field theories in flat space-time independence of the action from dimensional constants is related to scale and, more in general, conformal invariance. For diffeomorphisms invariant theories such as general relativity, this relationship becomes more involved [17]. In the case of Einstein gravity with a cosmological constant this scale symmetry can be easily identified as a subgroup of the isometry group of the de-Sitter spacetime, which is solution of the theory. We note in passing, that the dS/CFT (AdS/CFT) correspondence [19, 20] is also based on the conformal isometry of the de Sitter (anti de-Sitter) spacetime.

The scale symmetry is hidden in the action (11) because of the presence of the dimensional coupling constants $G, \lambda$, but becomes explicit in the action (31). Scale or more in general, conformal, symmetry has been already used in the past for trying to solve the cosmological constant problem [21, 22, 23, 24]. Usually one modifies the Einstein action, either by considering scalar-tensor theories or quadratic curvature terms, in order to construct a scale invariant action. Unfortunately, these modifications affect also the behavior of general relativity on distance scales where it works perfectly. In this paper we will not try to modify GR but we will select the regimes of the theory when conformal invariance manifests itself explicitly.

The investigation of the scale symmetry of the action (3) is rather complicated because of general covariance and the presence of many degrees of freedom. In particular, it is well known that scale invariance is not compatible with the general covariance of general relativity. The Einstein-Hilbert term $\sqrt{-g} R$ in the action (1I) is not scale invariant. In order to circumvent this problem we consider as in Ref. [15, 16] only conformally flat perturbation of the metric, i.e we fix the diffeomorphisms in the action and consider only one gravitational degree of freedom, the conformal factor of the metric:

$$
g_{\mu \nu}=\psi^{2} \eta_{\mu \nu}
$$

This is a rather drastic simplification, which we expect to apply only to configurations with a high degree of symmetry. But it allows to formulate a theory of gravity as a field theory in flat spacetime and makes evident the conformal properties of Einstein gravity coupled with a cosmological constant. Clearly, we pay a high price, we loose completely the general covariance of the theory and we throw away most of modes of the gravitational field. As a consequence we are forgetting the metric nature of the field $\psi$ and we are able to describe only a very limited part of gravitational physics. For instance, we cannot describe simple gravitational structures such as a black hole or global features of the spacetime such as cosmological horizons. On the other hand there are at least two regimes in which the gravitational field admits the description given by Eq. (4). The first is cosmology, a spatially flat Robertson-Walker universe. The second is the local behavior of the gravitational field. Locally, we can always turn off the gravitational field and 
set $g_{\mu \nu}=\eta_{\mu \nu}$. Obviously flat spacetime is not a exact solution of Einstein gravity with a cosmological constant. However, we can consider it as an approximate solution that holds when we consider gravitational physics at length scales $<<L_{\Lambda}=\Lambda^{-1 / 2}$. In this case the term $\Lambda g_{\mu \nu}$ in the Einstein equations can be neglected and flat spacetime becomes a solution of the theory. In terms of the ansatz (4) this behavior corresponds to an almost constant field $\psi$. It is also interesting to notice that our proposal is complementary to the so-called unimodular theories of gravity 25. In these theories the conformal factor of the metric is held fixed and the cosmological constant appears as an integration constant of the gravitational field equations.

Using Eq. (4) into the action (3), we get

$$
S=\frac{3}{8 \pi \alpha_{0}} \int d^{4} x\left[(\partial \psi)^{2}-\psi^{4}\right] .
$$

This is a CFT in flat space-time, which has the form of a $\lambda \phi^{4}$ field theory. Because we are fixing the diffeomorphisms of the action (31) we expect the appearance of constraints. In fact one can show, after some manipulation, that the Einstein equations stemming from the action (3) and evaluated with the ansatz (4) are equivalent to the field equations derived from (5) constrained by the equation

$$
T_{\mu \nu}^{(\psi)}=\psi\left(\partial_{\mu} \partial_{\nu} \psi-\frac{1}{4} \eta_{\mu \nu} \partial^{2} \psi\right)-2\left(\partial_{\mu} \psi \partial_{\nu} \psi-\frac{1}{4} \eta_{\mu \nu}(\partial \psi)^{2}\right)=0
$$

The tensor $T_{\mu \nu}^{(\psi)}$ can be interpreted as the improved stress-energy tensor of the 4D CFT defined by the action (5). In fact $T_{\mu \nu}^{(\psi)}$ is identically traceless and it is conserved, i.e satisfies $\partial_{\mu} T^{(\psi) \mu \nu}=0$, by virtue of the field equations

$$
\partial^{2} \psi+2 \psi^{3}=0
$$

coming from the action (5). We have therefore shown that the dynamics of the conformal factor of the metric in Einstein gravity with a cosmological constant can be described by a $4 \mathrm{D}$ CFT constrained by a vanishing stressenergy tensor. The action (5) is invariant under the action of the 4D conformal group generated by the infinitesimal transformations

$$
\xi^{\mu}=a^{\mu}+\omega_{\nu}^{\mu} x^{\nu}-\epsilon x^{\mu}+c^{\mu} x^{2}-2 x^{\mu} c_{\nu} x^{\nu}
$$

where $a^{\nu}, \omega_{\nu}^{\mu}, \epsilon, c^{\nu}$ generate respectively, translations, rotations, dilatations and special conformal transformations. The field $\psi$ transforms as a conformal field with weight -1 :

$$
\delta \psi=-\left(\xi^{\nu} \partial_{\nu}+\frac{1}{4}(\partial \xi)\right) \psi
$$

It is well know that the conformal symmetry puts stringent constraints on the form of the correlations functions of the theory. In particular, the solutions of the field equations (7) are $\psi=1 / \sqrt{\left(2 x^{2}\right)}$ (the so-called meron type solutions [17, 18]). Eq. (6) puts additional constraints on the form of the solution, so that only the solution $\psi=1 / t$ is allowed. Meron type solutions are an example of classical scale-free solutions. In the context of Einstein gravity with vanishing cosmological constant they have been used some time ago to argue about the short-distance independence of gravity from Newton constant [17].

The conformal symmetry of the gravitational sector is in general broken by coupling with matter fields $\chi^{M}$ and we expect that the dimensional coupling constants $G, \lambda$ are introduced by this breaking. The gravity-matter action becomes $S_{t o t}=S+S_{M}\left(\chi^{M}, g_{\mu \nu}\right)$, where $S$ is the gravitational action (1) and $S_{M}$ is the action for matter fields. Using Eq. (4) the action becomes

$$
S_{t o t}=\frac{3}{8 \pi \alpha_{0}} \int d^{4} x\left[(\partial \psi)^{2}-\psi^{4}\right]+S_{M}\left(\psi, \chi^{M}, \lambda\right)
$$

whereas the constraint (6) is now

$$
T_{\mu \nu}^{(\psi)}=-4 \pi G\left(T_{\mu \nu}^{M}-\frac{1}{4} \eta_{\mu \nu} T^{M}\right) \psi^{2},
$$

where $T_{\mu \nu}^{M}=T_{\mu \nu}^{M}\left(\psi, \chi^{M}, \lambda\right)$ is the usual matter stress-energy tensor (evaluated for the metric (4) ) and $T$ is its (flat spacetime) trace. The pattern of the conformal symmetry breaking depends on the form of the matter action 
$S_{M}\left(\psi, \chi^{M}, \lambda\right)$. In general we will have $S_{M}=S_{0}\left(\chi^{M}\right)+\lambda^{-2} \psi^{2} S_{1}\left(\chi^{M}\right)+\ldots \lambda^{-2 n} \psi^{2 n} S_{n}\left(\chi^{M}\right)+\ldots$ and corresponding terms in the stress-energy tensor $T_{\mu \nu}^{M}$.

The term $S_{0}$ corresponds to a conformally invariant matter action, which has $T^{M}=0$. In this case the field equations for $\psi$

$$
\partial^{2} \psi+2 \psi^{3}=\frac{4}{3} \pi G T^{M} \psi
$$

remain conformal invariant and it is the constraint (11) which breaks the symmetry and introduces in the theory the dimensional coupling $\alpha_{1}=\alpha_{0} \lambda^{-2}=G$ (unless the right hand side of Eq.(11) is identically zero). The symmetry breaking term $S_{1}$ introduces the coupling constant $\alpha_{1}$ in the field equations (12) and in the constraint (11). The term $S_{2}$ requires the introduction of the coupling constant $\alpha_{2}=\alpha_{0} \lambda^{-4}=G \lambda^{-2}$. Generically, the term $S_{n}$ introduces in the theory the coupling constant $\alpha_{n}=\alpha_{0} \lambda^{-2 n}=G \lambda^{-(2 n-2)}$. Notice that the coupling constants $\alpha_{0}, \alpha_{1} \ldots \alpha_{n}$ define a hierarchy of coupling constants of dimensionality $[L]^{0},[L]^{2}, \ldots[L]^{2 n}$. The terms introduced in the action by $\alpha_{n}$ dominate at large scales with respect to those introduced by $\alpha_{m}(m<n)$. Conversely, the terms introduced by $\alpha_{m}$ dominate at short scales. We therefore have scale-free, short-distance, gravitational physics characterized by $\alpha_{0}$. The intermediate (from terrestrial to astronomical distances) behavior breaks the conformal symmetry and is determined by $\alpha_{1}$. The long-distance (cosmological scales) behavior breaks also conformal symmetry and is characterized by the coupling constant $\alpha_{2}$.

We can easily apply our approach to cosmology. In fact in the case of a spatially flat Robertson-Walker universe the metric can be put in the form (4), just by introducing the conformal time $t$. The field $\psi$ describes the radius of the universe and is a function of $t$ only. For matter in the form of a perfect fluid characterized by pressure $p$ and density $\rho$ equations (12) and (11) become respectively

$$
\begin{aligned}
\ddot{\psi}-2 \psi^{3} & =-\frac{4}{3} \pi \alpha_{2}(3 p-\rho) \psi^{3} \\
\ddot{\psi}-2 \frac{(\dot{\psi})^{2}}{\psi} & =-4 \pi \alpha_{2}(p+\rho) \psi^{3} .
\end{aligned}
$$

The previous equation are equivalent to the usual Friedmann equations, we just need to change the time variable $d t=d t^{\prime} / \psi$ and to rescale $\psi$. Notice that the consistency condition between Equations (13) maintains the form it has for the Friedmann equations: $\dot{\rho}=-3(p+\rho)(\dot{\psi} / \psi)$.

Written in the form (13), the cosmological equations have a lot of interesting physical features which are completely obscured in the usual formulation. First of all, equations (13) depend on the coupling constant $\alpha_{2}$ but not on $\alpha_{1}=G$. This means that we are considering gravitation at (large) length scales where $G$ becomes irrelevant and the dynamics is determine completely by $G / \lambda^{2}$. At smaller length-scales (e.g solar system distances) gravitational physics is determined by $\alpha_{1}=G$ and $\alpha_{2}$ becomes irrelevant. Moreover, $\lambda$ appear in the equations as a coupling constant, in the same way as $G$. This solves the dichotomy mentioned at the beginning of this paper concerning the interpretation of $\Lambda$ both as a geometrical term independent of the distribution of matter and as a exotic form of matter. In our formulation the energy density driving the accelerating expansion of the universe can be considered just as a form of matter with equation of state $p_{a}=-\rho_{a}$. The cosmological constant $\lambda$ builds up with $G$ the strength $\alpha_{2}$ of its gravitational interaction.

The cosmological equations (13) also have a very nice physical interpretation. The first equation describes the dynamics of the conformal mode of the metric. This dynamics is conformally symmetric either when matter is absent $(p=\rho=0)$ or when the matter dynamics is also conformal $(p=\rho / 3)$. The second equation in (13), or equivalently Eq. (11), gives a relationship between the stress-energy of the conformal mode $\psi$ and the stress-energy tensor of matter. This equal footing description of sources and gravitational field is rather unusual in gravity theories. Using the consistency condition the same equation can be written as a general relation between the energy density of the conformal mode $\psi$ and the flux of matter:

$$
T_{00}^{(\psi)}=-3 \pi \alpha_{2}(p+\rho) \psi^{4}=\pi \alpha_{2} \frac{\psi^{5}}{\dot{\psi}} \dot{\rho} .
$$

For matter with equation of state $p=-\rho$ both the flux of matter and the energy density of the conformal mode are zero. For $p>-\rho(p<-\rho)$ both the flux of matter and the energy density of $\psi$ are negative (positive).

Let us now discuss the implication of our results for the cosmological constant problem. We have argued above that the gravitational field allows for a parametrization as in Eq. (4) in two regimes: its behavior on length scales $<<L_{\Lambda}$ and cosmology. These are two limiting regimes, but are the relevant ones for the cosmological constant problem. In 
fact this problem arises from the interplay between micro- and macro-physics. We do not expect that gravitational physics at intermediate scales - such as astronomical distances - could have some impact on the problem. If our description applies at short distances, the conformal symmetry could be responsible for driving to zero the vacuum energy of quantum fields. A mechanism to achieve this goal has been proposed in Ref. [16]. It is based on the quantum triviality of the $\lambda \phi^{4}$ theory that could drive to zero the value of the cosmological constant.

The constant energy density driving the accelerating expansion of the universe could be generated by the coupling with matter, which breaks the conformal invariance (see Eq. (11), (12)). More in detail this energy density is generated by the terms in the matter action that depend on $\psi^{4}$ and couple to gravity trough the coupling constant $\alpha_{2}$. Because, the origin of this energy density is the coupling with ordinary matter we naturally expect it to be of order $\rho^{(o b s)}$.

The key question to be answered now is: how much is our approach reliable? After all we are describing gravity in a very rough way and we are spoiling it by most of the nice features of the metric theories of gravity. Moreover, the mechanism proposed in Ref. [15, 16] that drives the cosmological constant to zero has more or less the status of a conjecture and should be made more precise. On the other hand our approach makes the conformal invariance of Einstein gravity with a cosmological constant explicit. Conformal invariance can be hardly reconciled with the general covariance of the theory. Some fixing of the diffeomorphisms invariance of GR is therefore required in order to make the scale symmetry explicit. It is obvious that these investigations have to be considered as a starting point for trying to build a theory of pure gravity that is manifestly scale invariant while maintaining most of the features of GR, at least at astronomical length scales. Because the main results of our paper are based on symmetry arguments we expect them to remain true for such would-be theory. In view of the above-mentioned incompatibility between scale symmetry and general covariance, the task of building such a theory is far from being trivial. It is likely that deep modifications of GR will be required.

Another point, which deserves interest is the physical origin of the coupling constant $\alpha_{2}$ (or equivalently $\lambda$ ) and of the energy density $\rho_{a}$ with equation of state $p_{a}=-\rho_{a} . \alpha_{2}$ is a free parameter, so that in order to determine both $\alpha_{2}$ and $\rho_{a}$ we need beside the observed value $\rho^{(o b s)}$ other independent observational data involving them. Of course this requires detailed information about the origin of of $\rho_{a}$. We just know that $\rho_{a}$ is generated by terms in the matter action depending on $\psi^{4}$. For instance such term can be generated by the potential of a scalar field coupled to gravity. More in general they could be generated by global effects of the gravitational field and/or of the quantum vacuum of matter fields for which our simplified description does not apply.

* Electronic address: mariano.cadoni@ca.infn.it

[1] S. Weinberg, Rev. Mod. Phys. 61 (1989) 1.

[2] S. M. Carroll, Living Rev. Rel. 4 (2001) 1 arXiv:astro-ph/0004075.

[3] T. Padmanabhan, Phys. Rept. 380 (2003) 235 arXiv:hep-th/0212290.

[4] P. J. E. Peebles and B. Ratra, Rev. Mod. Phys. 75 (2003) 559 arXiv:astro-ph/0207347.

[5] U. Ellwanger, arXiv:hep-ph/0203252

[6] S. Nobbenhuis, arXiv:gr-qc/0411093

[7] A. G. Riess et al. [Supernova Search Team Collaboration], Astron. J. 116 (1998) 1009 arXiv:astro-ph/9805201.

[8] S. Perlmutter et al. [Supernova Cosmology Project Collaboration], Astrophys. J. 517 (1999) 565 arXiv:astro-ph/9812133.

[9] C. B. Netterfield et al. [Boomerang Collaboration], Astrophys. J. 571 (2002) 604 arXiv:astro-ph/0104460.

[10] R. A. Knop et al. [The Supernova Cosmology Project Collaboration], Astrophys. J. 598 (2003) 102 arXiv:astro-ph/0309368.

[11] J. L. Tonry et al. [Supernova Search Team Collaboration], Astrophys. J. 594 (2003) 1 arXiv:astro-ph/0305008.

[12] D. N. Spergel et al. [WMAP Collaboration], Astrophys. J. Suppl. 148 (2003) 175 arXiv:astro-ph/0302209.

[13] M. Tegmark et al. [SDSS Collaboration], Astrophys. J. 606 (2004) 702 arXiv:astro-ph/0310725.

[14] A. G. Riess et al. [Supernova Search Team Collaboration], Astrophys. J. 607 (2004) 665 arXiv:astro-ph/0402512.

[15] A. M. Polyakov, Phys. Atom. Nucl. 64 (2001) 540 [Yad. Fiz. 64 (2001 IMPAE,A16,4511-4526.2001) 594] arXiv:hep-th/0006132.

[16] R. Jackiw, C. Nunez and S. Y. Pi, Phys. Lett. A 347 (2005) 47 arXiv:hep-th/0502215.

[17] V. de Alfaro, S. Fubini and G. Furlan, Nuovo Cim. B 57 (1980) 227.

[18] V. de Alfaro, S. Fubini and G. Furlan, Nuovo Cim. A 50 (1979) 523.

[19] A. Strominger, JHEP 0110 (2001) 034 arXiv:hep-th/0106113.

[20] J. M. Maldacena, Adv. Theor. Math. Phys. 2 (1998) 231 [Int. J. Theor. Phys. 38 (1999) 1113] arXiv:hep-th/9711200].

[21] A. Zee, Phys. Lett. B 109 (1982) 183.

[22] R. Booth, arXiv:gr-qc/0203065

[23] J. Barbour, Class. Quant. Grav. 20 (2003) 1543 arXiv:gr-qc/0211021.

[24] P. D. Mannheim, Found. Phys. 30 (2000) 709 arXiv:gr-qc/0001011. 
[25] W. G. Unruh, Phys. Rev. D 40 (1989) 1048.

[26] This two questions are referred to as the old cosmological constant problem. A third puzzling point with which we are faced when trying to understand the universe in which we live is the coincidence problem: Why is the vacuum energy density today of the same order of magnitude as that of matter? In this paper we will not address this question. 